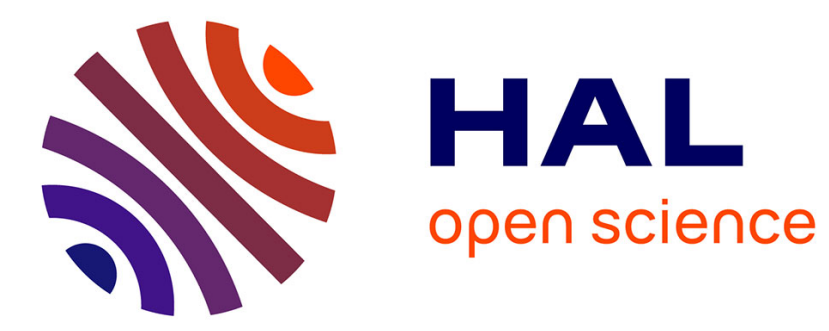

\title{
Two-step assembly kinetics of gold nanoparticles
}

\author{
Jieli Lyu, Damien Alloyeau, Cyrille Hamon, Doru Constantin
}

\section{To cite this version:}

Jieli Lyu, Damien Alloyeau, Cyrille Hamon, Doru Constantin. Two-step assembly kinetics of gold nanoparticles. Journal of Materials Chemistry C, 2021, 9 (5), pp.1730-1739. 10.1039/D0TC05076H . hal-03365388

\section{HAL Id: hal-03365388 \\ https://hal.science/hal-03365388}

Submitted on 5 Oct 2021

HAL is a multi-disciplinary open access archive for the deposit and dissemination of scientific research documents, whether they are published or not. The documents may come from teaching and research institutions in France or abroad, or from public or private research centers.
L'archive ouverte pluridisciplinaire HAL, est destinée au dépôt et à la diffusion de documents scientifiques de niveau recherche, publiés ou non, émanant des établissements d'enseignement et de recherche français ou étrangers, des laboratoires publics ou privés. 


\title{
Two-step assembly kinetics of gold nanoparticles
}

\author{
Jieli Lyu, ${ }^{\dagger}$ Damien Alloyeau, ${ }^{\ddagger}$ Cyrille Hamon, ${ }^{*} \dagger$ and Doru Constantin ${ }^{*, \dagger}$ \\ †Université Paris-Saclay, CNRS, Laboratoire de Physique des Solides, 91405 Orsay, France \\ $\ddagger$ Laboratoire Matériaux et Phénomènes Quantiques, Université de Paris - CNRS, F-75013, \\ Paris, France
}

E-mail: cyrille.hamon@universite-paris-saclay.fr; doru.constantin@universite-paris-saclay.fr

\begin{abstract}
We study the assembly kinetics of surfactant-stabilized gold nanoparticles in the presence of sulfate ions. The reaction proceeds in two steps: very rapid (a few minutes) formation of amorphous aggregates, followed by slow reordering (over several hours). The latter process is the only one detectable via absorbance spectroscopy and results in the formation of intimate contacts between the objects, with interparticle distances below the thickness of a surfactant bilayer. The rate-limiting step of the reaction could be related to surfactant expulsion from the initial aggregates, which allows the particles to come in close contact and form chains. There are marked differences in reaction yield and rate constant between spheres, rods and bipyramids, highlighting the role of surface curvature in contact formation. Once formed, the assemblies are very sturdy and stable under centrifugation and dialysis. The contact interaction is strong and highly directional, as shown by liquid-cell transmission electron microscopy.
\end{abstract}

\section{Introduction}

Gold nanoparticles (GNPs) are exciting nanoscale building blocks, due to their intrinsic properties (such as the surface plasmon resonance) which are useful in applications, but 
also due to the way these properties vary when the particles are brought close together, yielding materials with emergent properties that can be very different from those of the isolated objects. GNPs are easily assembled into compact structures, e.g. by van der Waals or depletion forces, but it is much more difficult to obtain open or elongated patterns. Endto-end assembly into long chains (plasmonic polymers) is of particular interest, ${ }^{1}$ since the longitudinal plasmon peak of the aggregate can be red-shifted over a very wide wavelength range, depending on the number of units in the chain and on the tip-to-tip distance.

Plasmonic polymers have been obtained by wet chemical approaches, ${ }^{2}$ including but not limited to molecular recognition ${ }^{3-8}$ or solvophobic interaction ${ }^{9,10}$ or mediated by templates. $^{11}$ In many cases, the self-assembly reaction progresses until flocculation and can be difficult to handle. In this respect, it is crucial to control the assembly process and stabilize a preferred configuration with defined optical properties. This requires the ability to controllably manipulate the reaction rate, which can be achieved by an external stimulus. A light-controlled synthetic procedure has been demonstrated, but this technique necessitates a femtosecond laser tuned to the plasmon frequencies of the nanoparticles. ${ }^{12-14}$ Benchtop methods relying on purely chemical strategies have been developed to prevent the growth of larger structures such as a molecular competitor ${ }^{12,15}$ (e.g. addition of an excess of mono-thiol to compete a dithiolated linker mediated assembly) or by mean of encapsulating the oligomers. ${ }^{16,17}$ In these cases, the surface of the oligomers as well as the particle junctions are passivated by strongly bound ligands, which may impede further use. This shortcoming can be avoided by a simpler strategy, which involves adding salt to a particle suspension in various solvents, such as DMF, ${ }^{18}$ ethanol $^{19}$ or a DMF-water mixture. ${ }^{20}$

In a recent study, sulfate ions were used to self-assemble gold nanorods into chains in water. ${ }^{21}$ We adapted this procedure to gold bipyramids and found that the self-assembly process can be stopped at any stage by adding excess surfactant to the reaction mixture. ${ }^{22}$ We also explored the optical properties of the chains after engineering conductive junctions using various metals. 
In the present work, various types of surfactant-stabilized GNPs (spheres, rods and bipyramids) were self-assembled using sulfate ions. We investigated the reaction kinetics by UV/Vis absorbance spectroscopy (AS) and small angle X-Ray scattering (SAXS) and found a two-step mechanism: (1) an initial fast aggregation process (detected by SAXS), in which particles are loosely bound and (2) a slow self-assembly step whereby the particles come into intimate contact. During this step, the AS spectrum of the GNPs changes, and we analyze it quantitatively to extract the time-dependent fraction of monomers and chains as well as the short-scale structure of the latter. Finally, the chain size and structure was also studied by ex situ and liquid cell transmission electron microscopy (TEM and LCTEM, respectively).

\section{Materials and Methods}

\section{Particle synthesis}

All the starting materials were obtained from commercial suppliers and used without further purification: Hexadecyltrimethylammonium bromide (CTAB, $\geq 99 \%$ ), hexadecyltrimethylammonium chloride (CTAC, 25 wt\% in $\mathrm{H}_{2} \mathrm{O}$ ), 5-bromosalicylic acid (90\%), hydrogen tetrachloroaurate trihydrate $\left(\mathrm{HAuCl}_{4} \cdot 3 \mathrm{H}_{2} \mathrm{O}, \geq 99.9 \%\right)$, silver nitrate $\left(\mathrm{AgNO}_{3}, \geq 99 \%\right)$, Lascorbic acid $(\geq 99 \%)$, sodium borohydride $\left(\mathrm{NaBH}_{4}, 99 \%\right)$ were purchased from Sigma Aldrich. Magnesium sulfate $\left(\mathrm{MgSO}_{4}, \geq 95 \%\right)$ was purchased from Prolabo. Water purified by reverse osmosis with a resistivity above $15 \mathrm{M} \Omega . c m$ was used in all experiments.

Gold nanoparticles (GNPs) were synthesized through seed-mediated approaches according to previously reported methods. More specifically, monocrystalline gold nanorods were obtained by a synthetic method involving 5-bromosalicylic acid as co-reducing agent. ${ }^{23,24}$ Gold bipyramids were prepared with a high yield following recently published protocols in which pentatwinned seeds are used. ${ }^{25,26}$ Nanospherse were prepared by two growth steps onto monocrystalline seeds. ${ }^{27}$ After synthesis, suspension were purified from the excess of 
reactant by 4 centrifugations (at $7100 \mathrm{~g}$ for $40 \mathrm{~min}$ ) and pellet redispersion in a CTAB solution for final concentrations of $15.6 \mathrm{mM} \mathrm{Au}{ }^{0}$ and $1.8 \mathrm{mM}$ CTAB. Once prepared suspension were stable for months.

\section{Particle assembly}

In a typical experiment, $16 \mu \mathrm{L}$ of the stock GNP suspension (at $15.6 \mathrm{mM} \mathrm{Au}{ }^{0}$ and $1.8 \mathrm{mM}$ CTAB) were pipetted into a vial or cuvette, followed by a variable amount (a few tens of $\mu \mathrm{L}$ ) of a $1.8 \mathrm{mM}$ CTAB solution and by $1 \mathrm{~mL}$ of a $1 \mathrm{mM} \mathrm{MgSO}_{4}$ solution. Note that the order of mixing is important: premixing the $\mathrm{CTAB}$ and $\mathrm{MgSO}_{4}$ solutions before adding them to the stock GNP suspension often leads to rapid (a few s) precipitation of the particles, revealed by a grayish color of the solution.

The polymerization rate can be modulated by changing the CTAB concentration in the mixture. At any time of the reaction course, $13.25 \mu \mathrm{L}$ of a $0.756 \mathrm{M}$ CTAC or CTAB solution can be added to the mixture in order to stop the assembly.

For $\mathrm{AS}$, the mixture was prepared in a $1 \mathrm{~mL}$ polystyrene cuvette and inserted within 30 seconds in the instrument to start recording.

For SAXS, we filled the solutions into round glass capillaries, with an outer diameter of $1.2 \mathrm{~mm}$ and 10 micron-thick walls, purchased from WJM-Glas (Berlin, Germany), placed vertically in a motorized and temperature-controlled holder. The capillaries were sealed at the top to prevent evaporation. The measurements were performed at room temperature $\left(22^{\circ} \mathrm{C}\right)$.

\section{SAXS}

The SAXS measurements were performed on the SWING beamline of the SOLEIL synchrotron (Saint-Aubin, France). The beam size was approximately $500 \times 200 \mu \mathrm{m}^{2}(H \times V)$. The scattered signal was recorded by an Eiger 4M detector (Dectris Ltd., Switzerland) with pixel size $75 \mu \mathrm{m}$. Preliminary data treatment (angular averaging and normalization) was 
done using the software Foxtrot developed at the beamline and yielded the intensity as a function of the scattering vector $I(q)$ in absolute units.

Subsequent data analysis was done in Igor Pro using models available in the NCNR

SANS package. ${ }^{28}$ Specifically, we determine the (isotropically averaged) form factor $F(q)$ of the particles by measuring a well-dispersed solution and obtain an approximate structure factor as $S(q)=I(q) / F(q)$.

The "fast" experiment (1h total time) was performed at a sample-to-detector distance of $6.03 \mathrm{~m}$, with a photon energy $E=16 \mathrm{keV}$. For the "slow" experiment (18h total time) the sample-to-detector distance was $6.52 \mathrm{~m}$ and the photon energy was $E=12 \mathrm{keV}$.

\section{UV-Vis-NIR absorbance spectroscopy}

We used a Cary 5000 spectrometer (Agilent) in a dual-beam configuration with a waterfilled cuvette in the reference path to measure the absorption spectrum of dilute particle solutions between 200 and $1200 \mathrm{~nm}$. The solutions were held in $1 \mathrm{~mL}$ polystyrene cuvettes with $1 \mathrm{~cm}$ optical path. The spectra were analyzed using the singular value decomposition (SVD) method, as detailed in the Supporting Information. Simulated extinction spectra were calculated by the boundary element method (BEM), using the MNPBEM toolbox developed in the MATLAB environment. ${ }^{29}$

\section{Transmission electron microscopy}

Conventional ex situ transmission electron microscopy (TEM) images were obtained with a JEOL 1400 microscope, operating at an acceleration voltage of $120 \mathrm{kV}$. Before observation, the solutions were at an $\mathrm{Au}^{0}$ concentration of $0.25 \mathrm{mM}$ in $10 \mathrm{mM}$ CTAC. We diluted them in a $0.2 \mathrm{mM}$ CTAC solution and concentrated the NPs by mild centrifugation at $2300 \mathrm{rpm}$ to a final $\mathrm{Au}^{0}$ concentration of $1.66 \mathrm{mM}$. Solution drops were then deposited on carbon coated grids and dried at $70^{\circ} \mathrm{C}$ to limit particle aggregation during the drying process.

The distribution of particle assemblies was obtained using the ImageJ software. We 
determined the average area of the isolated particles and used it to normalize the area of all identified objects. The objects were sorted according to the normalized area and the number of chains with $n$ monomers was calculated by summing objects between $n-0.5$ and $n+0.5$. The contact distribution was obtained by manually counting all the contacts in 5 images.

\section{LCTEM}

Liquid STEM imaging was performed on a JEOL 2100+ microscope operating at $200 \mathrm{kV}$, using a high angle annular dark field detector (STEM-HAADF) and a liquid-cell TEM holder (Poseidon select, Protochips inc.). A colloidal suspension of gold bipyramidal seeds (with an $\mathrm{Au}^{0}$ concentration of $2.5 \mathrm{mM}$ ) dispersed in a CTAC aqueous solution (10 mM) was encapsulated in the liquid-cell by using the conventional loading process. ${ }^{30,31}$ The Au spacers of the liquid cell were $150 \mathrm{~nm}$ thick and the experiments were performed in the corners of the observation window, where the liquid thickness is minimum. The liquid cell was used in static mode. Videos were acquired with a frame rate of 25 frames per second.

From each movie we extract every third frame and determine the outlines of the two particles using the ImageAnalyzeParticles operation in Igor Pro 7.0 (we discard the frames where the algorithm identifies more or less than two objects). The useful information is the position of the center of mass and the orientation of the objects, given by the angle of the major axis for the fitted ellipse. In the Videos, the center of mass is indicated by a red dot and the ellipse is drawn in blue.

\section{Results and Discussion}

\section{Particle shapes}

The diameters $D$ for all particles (obtained by SAXS) and the aspect ratios $A R$ (obtained by AS or TEM, as indicated) for rods and bipyramids are given below:

$$
\text { Spheres : } D=19 \pm 1 \mathrm{~nm}
$$


Rods: $D=17 \pm 4.5 \mathrm{~nm}, A R=2.9 \pm 0.13$ (AS) or $2.8 \pm 0.4(\mathrm{TEM})$.

Bipyramids: $D=17.5 \pm 2.5 \mathrm{~nm}, A R=2.6 \pm 0.2$ (TEM).

All parameters above are given as value \pm standard deviation (SD). The mean value of the $A R$ was obtained by comparison with Boundary Element Method (BEM) simulations, while its standard deviation was extracted by fitting the spectrum with an analytical model. ${ }^{32}$ The TEM histograms and the AS data and fits are available in the Supplementary Information (Figures S1 and S2).

\section{Following the kinetics by TEM}

We present in Figure 1 TEM images taken at the beginning $(0 \mathrm{~h})$ and the end $(20 \mathrm{~h})$ of the assembly process for the three particle shapes. In the right column we zoom in on individual chains.

a)

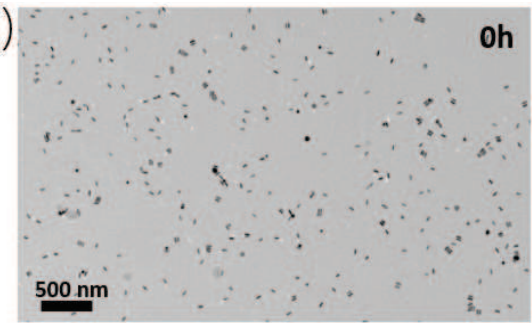

b)

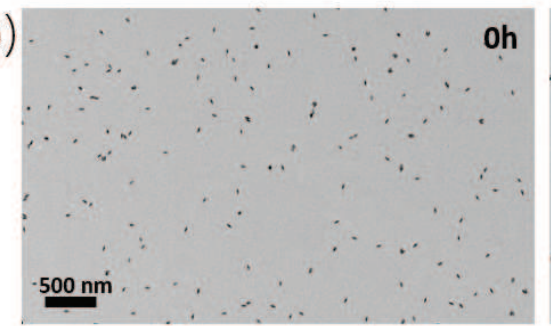

c)

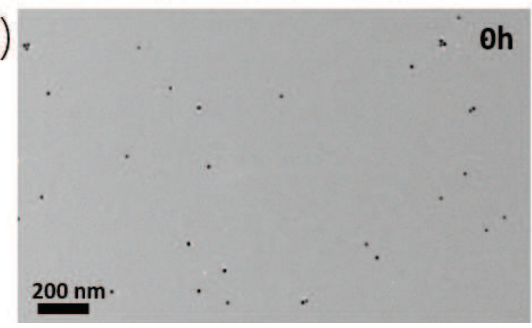

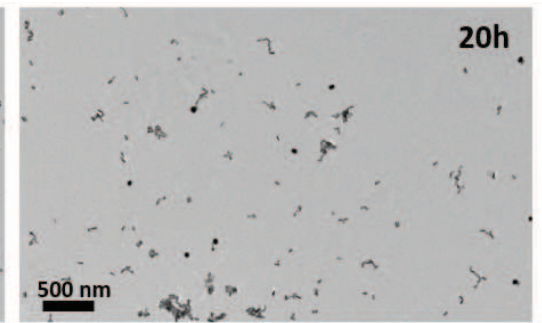
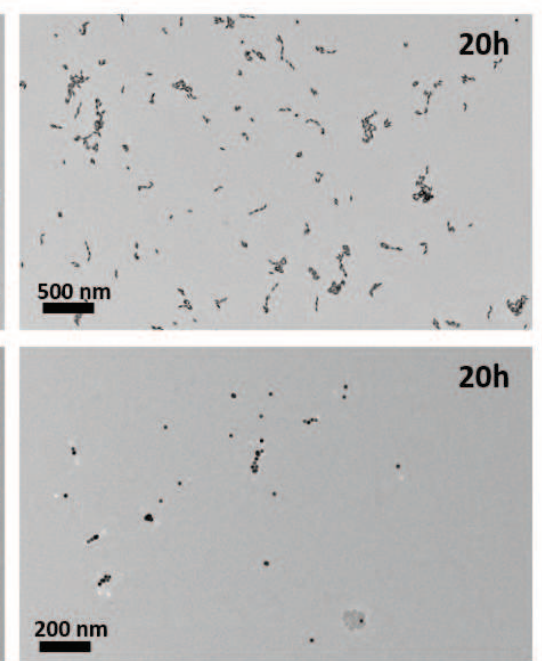
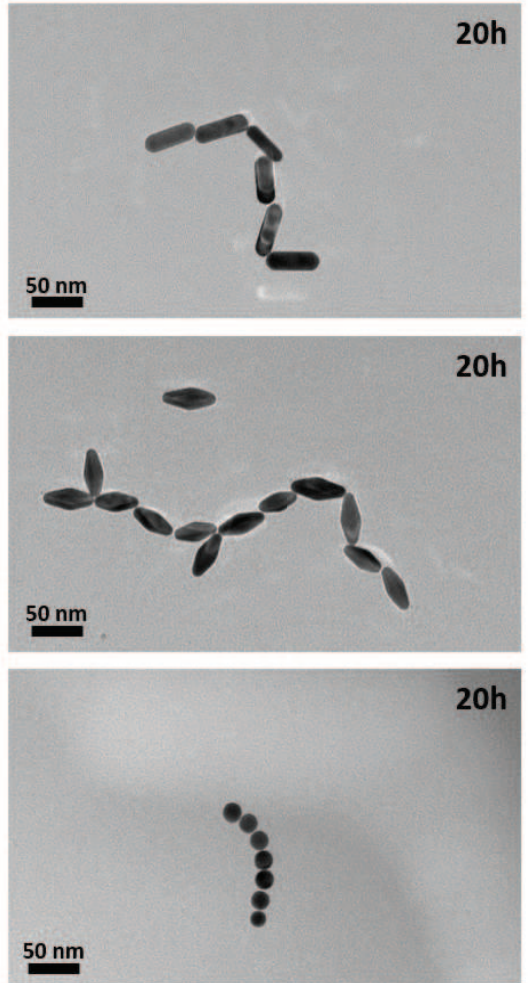

Figure 1: TEM images of the assembly state at $0 \mathrm{~h}$ and $20 \mathrm{~h}$. a) GNRs, b) GBPs and c) GNSs. The rightmost panel shows individual chains under higher magnification. 


\section{First step: fast aggregation due to surfactant dilution}

As we shall see, the aggregation only occurred in the presence of sulfate and if the CTAB concentration was low enough, so we studied the effect of both these parameters by preparing four gold nanorod solutions with and without $\mathrm{MgSO}_{4}$, at high and low CTAB concentration, while keeping the gold concentration fixed (Figure 2).
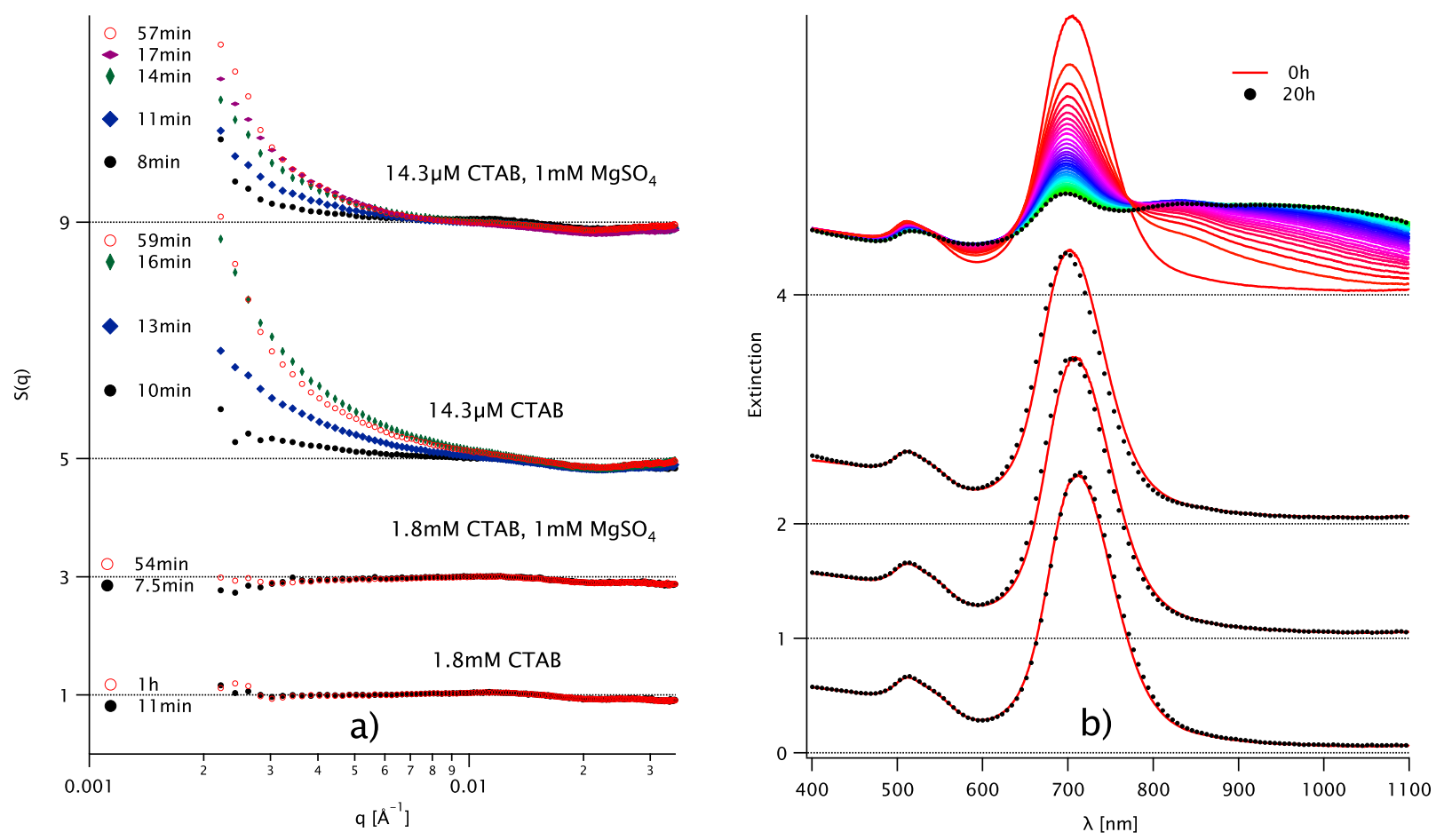

Figure 2: a) SAXS structure factors of nanorods at CTAB and $\mathrm{MgSO}_{4}$ concentrations indicated on top of the curves, for various times after preparation (different colors and markers). b) Extinction spectra for the solutions in a) immediately after preparation and after $20 \mathrm{~h}$. For the solution at $14.3 \mu \mathrm{M} \mathrm{CTAB}$ and $1.0 \mathrm{mM} \mathrm{MgSO}_{4}$, intermediate spectra (taken every 30 min.) are also shown. Curves shifted vertically for clarity: SAXS and corresponding AS data for the same solution are at the same level.

We followed the resulting solutions by SAXS (over $1 \mathrm{~h}$ ) and by AS (over $20 \mathrm{~h}$ ), as shown in Figure 2.

At high CTAB concentration, the structure factor $S(q)$ (Figure 2a) remained flat, indicating well-dispersed particles. At low CTAB concentration, 8 to 10 min after preparation there was already an increase in $S(q)$ at low $q$, sign that the particles aggregated. The aggregation process continued until about $20 \mathrm{~min}$ and then there was no change until the 
end of the experiment (1h). Note that the presence of $\mathrm{MgSO}_{4}$ had no effect on the SAXS results. We conclude that the first step of the assembly process is fast aggregation due to the reduction in surfactant concentration.

\section{Second step: slow assembly due to the presence of sulfate}

The AS data (Figure 2b) at high CTAB concentration showed no change over $20 \mathrm{~h}$, confirming good particle dispersion. Although no AS change occured at low CTAB concentration and in the absence of $\mathrm{MgSO}_{4}$, the $\mathrm{SAXS}$ data clearly shows the particles were aggregated. It is only when both conditions (low CTAB and presence of $\mathrm{MgSO}_{4}$ ) are met that the assembly occurred, as seen by the gradual changes in the spectrum (to be discussed in detail below).

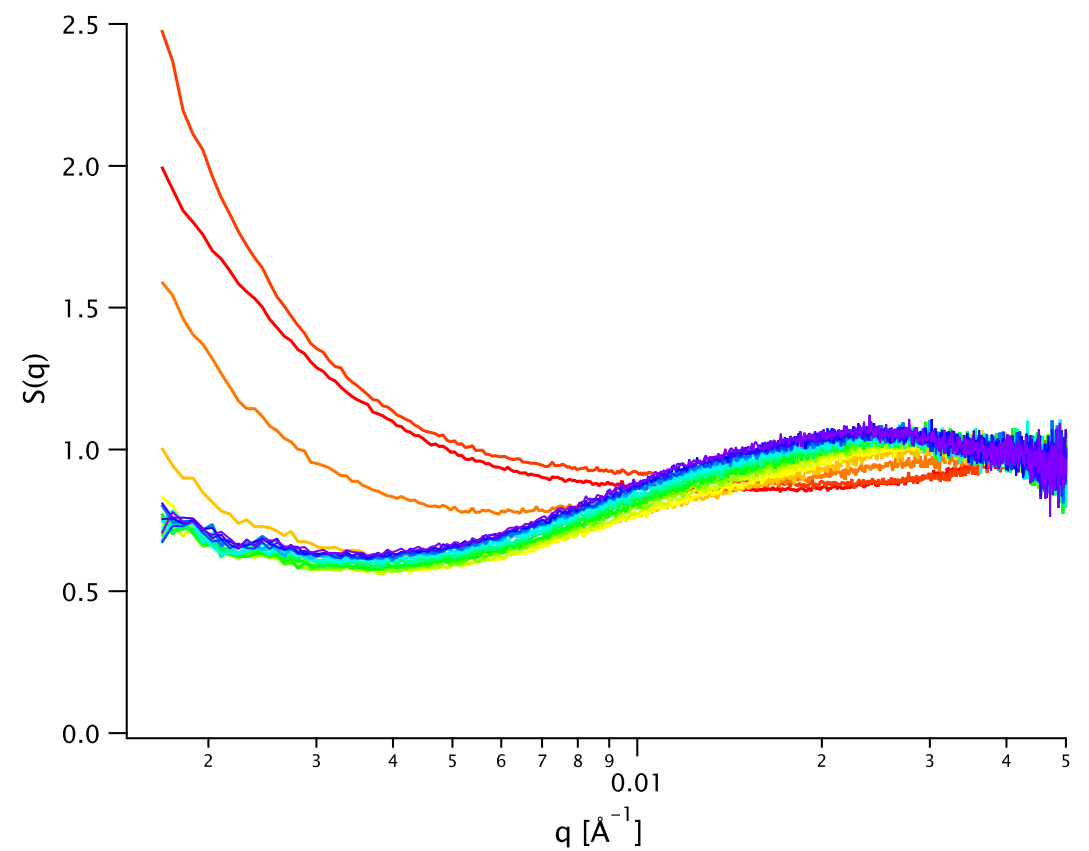

Figure 3: Assembly kinetics for nanorods with $1.0 \mathrm{mM} \mathrm{MgSO}_{4}$ and $28 \mu \mathrm{M}$ CTAB. SAXS curves acquired at 30 min intervals and color-coded from red to violet.

To get more insight into the structure of the assemblies, we performed a longer $(20 \mathrm{~h})$ TR-SAXS study (see Figure 3) for a sample in appropriate conditions (0.22 mM Au, $1.0 \mathrm{mM}$ $\mathrm{MgSO}_{4}$ and $\left.28 \mu \mathrm{M} \mathrm{CTAB}\right)$. We conducted the assembly reaction for the different nanoparticle morphologies and monitored the process by time-resolved AS (One representative examples 
is shown in Figure 4). For all particle shapes new absorbance bands appeared in the spectra, red-shifted with respect to the initial ones, indicating the formation of close contacts between particles. In the case of spheres the plasmon band centered at $525 \mathrm{~nm}$ decreased slightly, while a moderately intense band appeared at $700 \mathrm{~nm}$. In contrast, the spectra of rods and bipyramids varied significantly under otherwise similar experimental conditions. Their transverse band around $510 \mathrm{~nm}$ did not change noticeably, but the longitudinal plasmon band decreased in intensity and a new red-shifted broad band appeared. This evolution is an indication of particle assembly in a tip-to-tip configuration, ${ }^{33,34}$ that we confirmed by TEM (see Figure 1).

In the literature, the time evolution of the spectra is typically analyzed in terms of peak amplitude or area. This approach provides the typical time scale of the process, but not the yield, and it relies on subjective identification of the various modes. Here, we use SVD to extract the spectral signature of the relevant species and their time evolution (see Figure 4).

Using SVD, the complex, time-dependent extinction signal $A(\lambda, t)$ can be decomposed as a linear combination of $K$ components $A(\lambda, t)=\sum_{i=1}^{K} \mathbf{C}_{i}(t) \mathbf{S}_{i}(\lambda)$, where the $\mathbf{S}_{i}(\lambda)$ are the spectra of the various species and the $\mathbf{C}_{i}(t)$ are their time-dependent normalized concentrations, i.e. the fraction of monomers contained in objects of species $i$. Monomer number conservation requires $\sum_{i=1}^{K} \mathbf{C}_{i}(t)=1$ at all times. $\mathbf{C}_{i}(\infty)=\eta_{i}$ is the yield of species $i$.

Guided by the presence of isosbestic points (where the signal remained constant in time), we infer that only two components contributed significantly to the experimental spectra. ${ }^{35}$ Indeed, in terms of the formalism presented in the SI, singular values $\mathbf{W}_{i i}$ with $i>2$ were much smaller than the first two and we could describe the data very well using only $K=2$ components (see Figure $4 \mathrm{~b}$ and c for an example). The time dependence of the $\mathbf{C}$ coefficients was well described by an exponential:

$$
\mathbf{C}_{2}(t)=\mathbf{C}_{2}(\infty)[1-\exp (-k t)]
$$



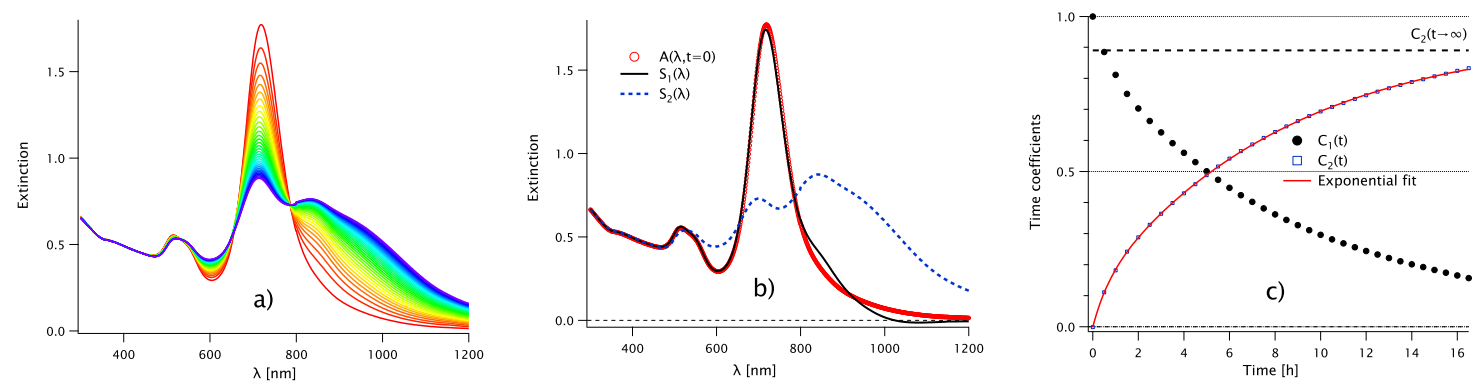

Figure 4: a) Time evolution of the extinction spectrum corresponding to the kinetics followed by SAXS in Figure 3, with the same time spacing and color code. b) SVD-extracted spectra of the first and second species, $\mathbf{S}_{1}(\lambda)$ and $\mathbf{S}_{2}(\lambda)$ (black solid line and blue dashed line, respectively). The first experimental spectrum (red open dots) is shown for comparison with $\mathbf{S}_{1}(\lambda)$. c) Time coefficients $\mathbf{C}_{1}(t)$ and $\mathbf{C}_{2}(t)$ of the identified species (black solid dots and blue open squares) with an exponential fit (red solid line). The limiting value $\mathbf{C}_{2}(\infty)$ is indicated as dashed line.

where the yield $\eta \sim \eta_{2}=\mathbf{C}_{2}(\infty)$ is the particle fraction that belongs to the aggregates (defined as objects characterized by $\mathbf{S}_{2}(\lambda)$ ) at the end of the process and .

\section{The role of $\mathrm{Au}$ concentration and the reaction order}

The concentration of reactant (in our case, the initially isolated GNPs) $c_{\mathrm{Au}}$ is a very important parameter, and its influence on the kinetics can reveal the reaction mechanism. We varied it by over an order of magnitude, from 0.0163 to $0.3 \mathrm{mM}$ and treated the AS data using SVD, as in Figure 4. The exponential dependence (1) is characteristic of an apparent first-order reaction: a reciprocal dependence (typical for second-order reactions) yields much worse fits, as shown in Figure S3 and Figure S4. The values of the yield and rate constant are shown in Figure 5. The yield decreased with $c_{\text {Au }}$ by about $30 \%$ over the studied range, while the rate constant was virtually unchanged, supporting our conclusion that the reaction is apparently first-order in the gold concentration, in disagreement with Ref. 21, but in agreement with other colloidal chain assembly processes, e.g. mediated by mercaptoethanol. ${ }^{36}$ We emphasize that this only applies to the second step of the process. Presumably, the rapid first step is collective in nature so we would expect it to have higher-order kinetics, but we could not test this hypothesis with the techniques we used. 

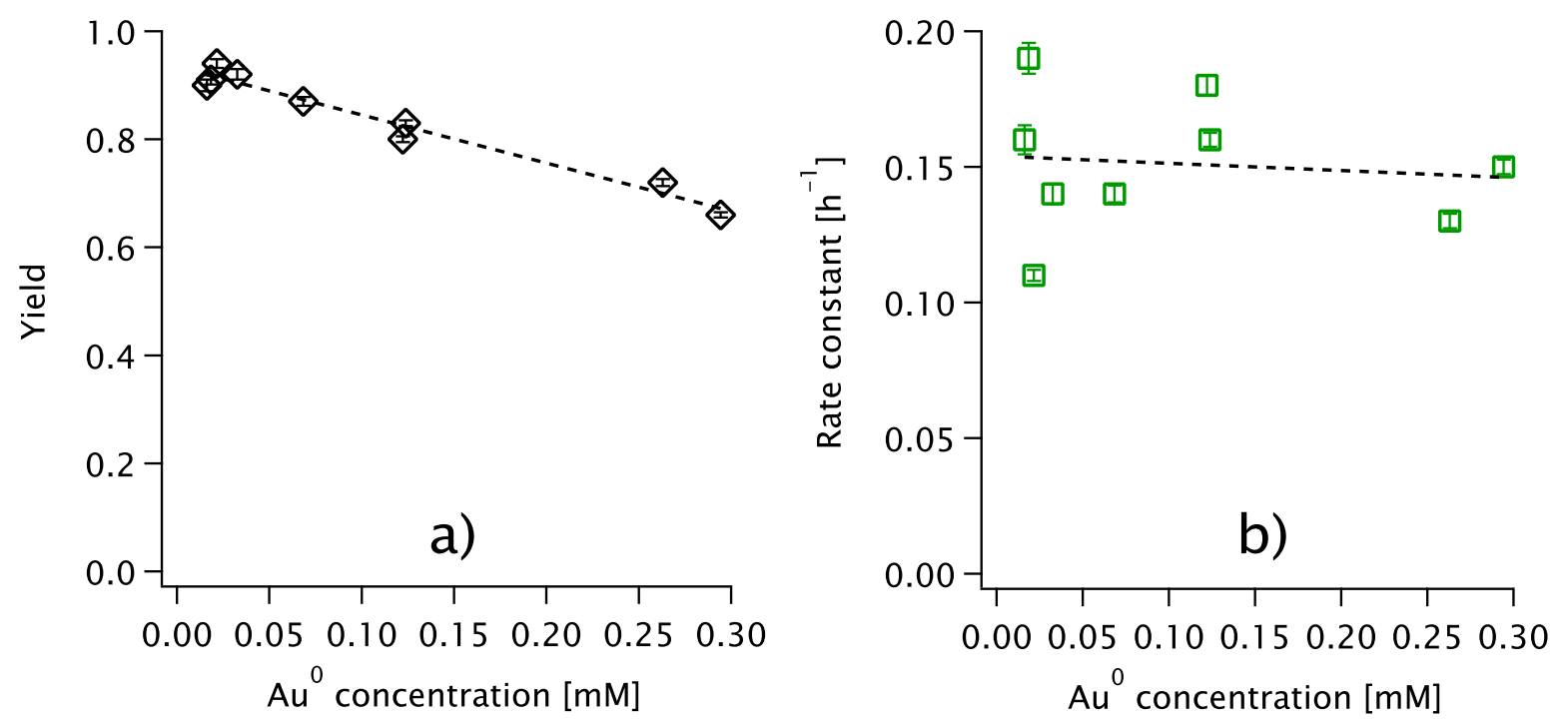

Figure 5: a) Yield and b) reaction rate constant for nanorod assembly as a function of $c_{\mathrm{Au}}$ in the presence of $1.0 \mathrm{mM} \mathrm{MgSO}_{4}$ and $14.3 \mu \mathrm{M}$ CTAB. Dashed lines are linear fits to the data.

\section{The role of sulfate}

The presence of sulfate ions is necessary for the formation of chains. We studied the kinetics for three different $\mathrm{MgSO}_{4}$ concentrations; the values of the yield and rate constant are shown in Figure 6. The yield was roughly linear in the $\mathrm{MgSO}_{4}$ concentration, which did not affect the rate.

\section{The role of surfactant}

Both the yield $\mathbf{C}_{2}(\infty)$ and the reaction rate constant $k$ are shown in Figure 7 as a function of the CTAB concentration in the presence of $1 \mathrm{mM} \mathrm{MgSO}_{4}$.

From these results, it is clear that the self-assembly yield increases as the CTAB concentration decreases. This experiment confirms that sulfate ions mediate the self-assembly of $\mathrm{GNPs}^{21}$ but also highlights the role of surfactant concentration in this process. Decreasing the CTAB concentration in solution well below the CMC (1 mM) ultimately leads to disruption of the bilayer at the surface of the NPs, weakening their electrostatic repulsion and rendering their surface accessible for conjugation with sulfate moieties. Moreover, the 

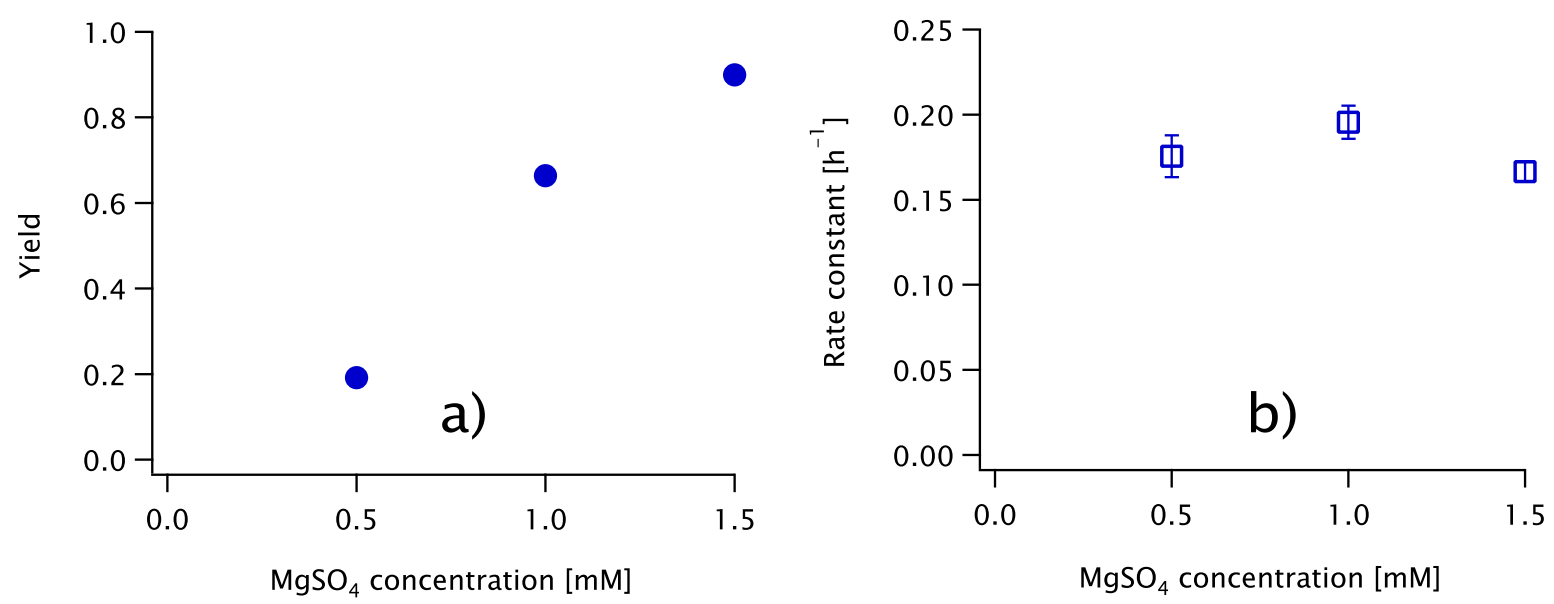

Figure 6: a) Yield and b) reaction rate constant for nanorod assembly as a function of the $\mathrm{MgSO}_{4}$ concentration for $c_{\mathrm{Au}}=0.25 \mathrm{mM}$ in the presence of $14.3 \mu \mathrm{M} \mathrm{CTAB}$.

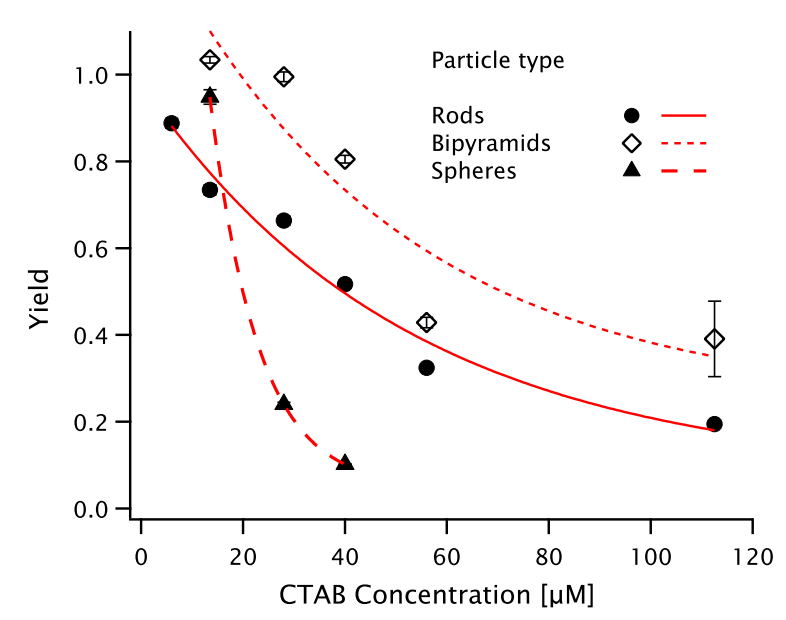

a)

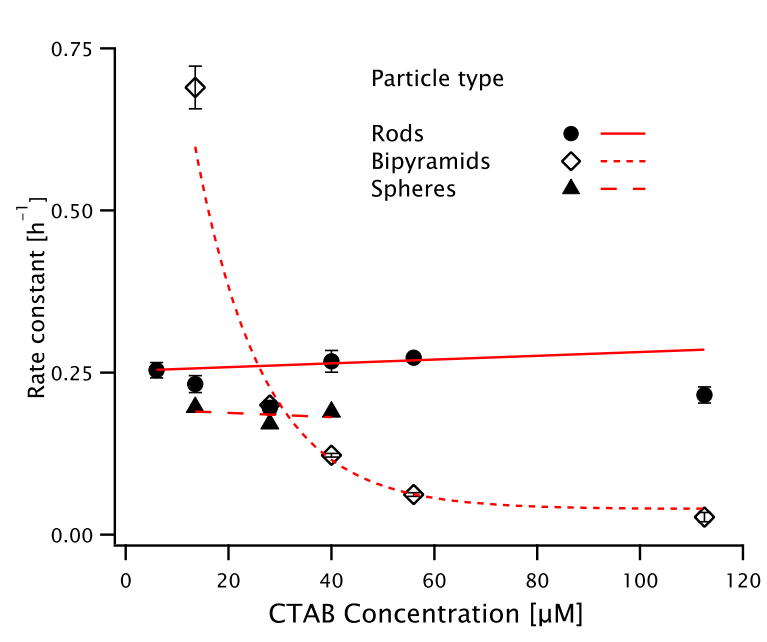

b)

Figure 7: a) Yield and b) reaction rate constant for particle assembly as a function of the CTAB concentration for $c_{\mathrm{Au}}=0.25 \mathrm{mM}$ in the presence of $1 \mathrm{mM} \mathrm{MgSO}_{4}$. The symbols correspond to different particle shapes. The lines are guides to the eye. 
CTAB density is lower at the tips of anisotropic NPs due to the higher local curvature compared to the lateral facets. ${ }^{37}$ Note that this explanation is qualitatively different from that of Abtahi et al., ${ }^{21}$ who invoke the association of sulfate ions with the head groups of CTAB. As discussed below, there is not enough place between the particle tips for two surfactant bilayers.

Our explanation entails that various particle shapes, with different curvature, should evolve differently. This is indeed the case, as one can see in Figure 7: the yield increased with the curvature (from spheres to rods to bipyramids). As expected, the reaction rate constant of nanorods was higher than that of spheres, but that of bipyramids showed a significantly different trend, decreasing steeply with the CTAB concentration. This might indicate a different mechanistic pathway, which requires further study to elucidate.

\section{Properties of the final state}

\section{Chain spectra and modelling}

One major advantage of the SVD technique is that it yields the spectra $\mathbf{S}_{2}(\lambda)$ of the final assemblies using a minimal number of assumptions. These spectra contain information on the inner structure of the assemblies, in particular on the contact configuration (tip-to-tip, side-to-side etc.) and on the interparticle distance at contact. In the case of nanorods, they are shown in Figure 8 for various CTAB concentrations.

Aided by BEM simulations (see Figures S6 to S11), we describe these spectra in terms of four contributions, highlighted by gray shading in the Figure. In order of increasing wavelength, they are:

1. The transverse band $(500-520 \mathrm{~nm})$ is not modified with respect to that of the isolated nanorods.

2. A narrow peak around $660 \mathrm{~nm}$, slightly blue-shifted from the longitudinal band of the isolated nanorods is a signature of side-to-side assembly (often observed in the TEM 


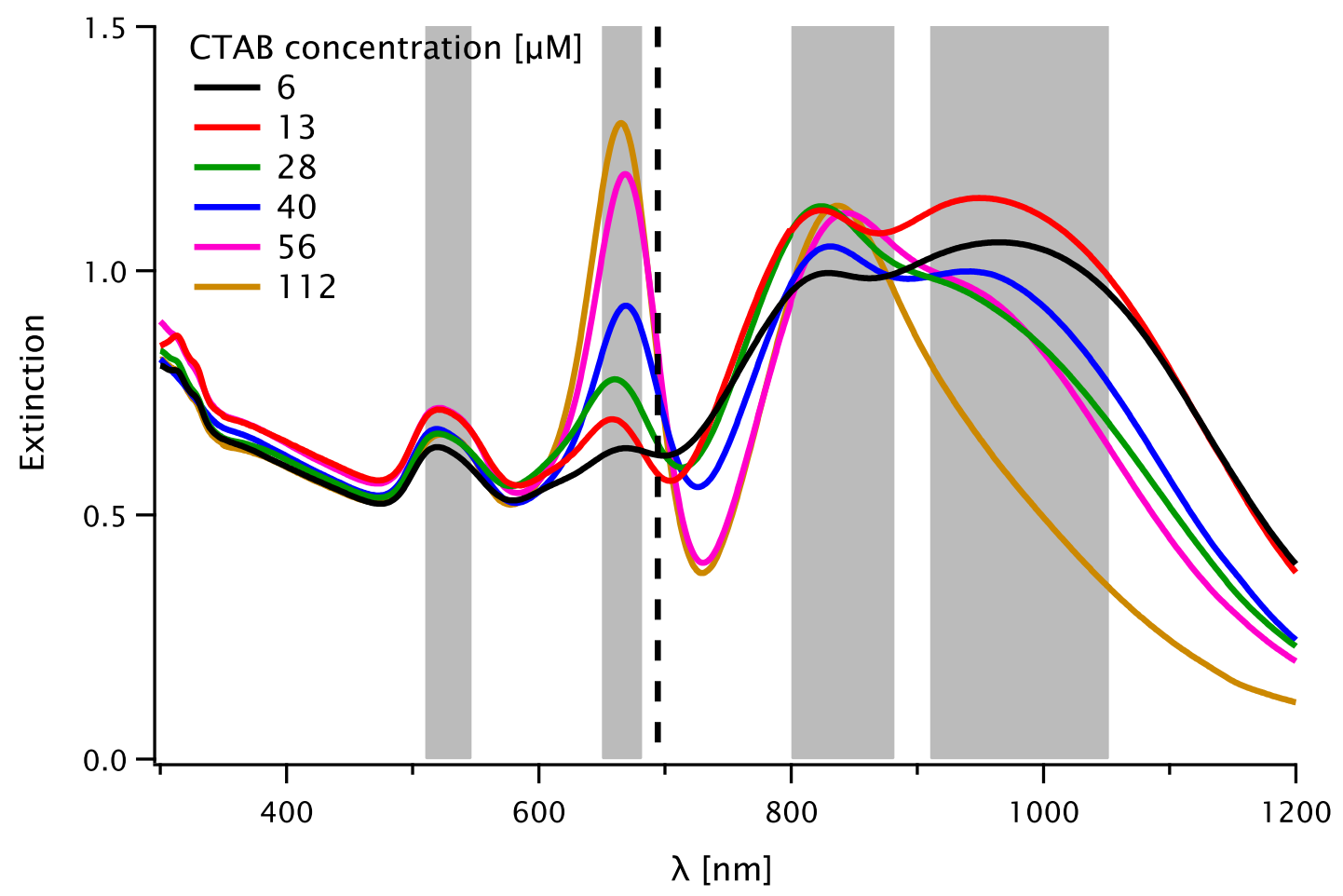

Figure 8: Extinction spectra $\mathbf{S}_{2}(\lambda)$ of the nanorod aggregates, for various CTAB concentrations (different colors). The position of the longitudinal peak for the isolated particle is shown as dashed line. Relevant plasmon bands are marked by gray shading (see text). 
images); from the amplitude of this shift we infer that the interparticle distance was between 6 and $10 \mathrm{~nm}$ (see the SI for more details), enough to accommodate two CTAB layers, with a thickness of $3.2 \mathrm{~nm} .{ }^{38,39}$ The amplitude of this peak increased with the CTAB concentration.

3. Two wide and superposed modes, centered around 850 and $950 \mathrm{~nm}$, respectively. Both are significantly red-shifted with respect to the longitudinal band of the isolated nanorods, marking the presence of even more anisotropic objects. We assign them to tip-to-tip chain assemblies (very frequently observed in the TEM images). The red shift depends on the number of particles in the chains, but also, very sensitively, on the tip-to-tip distance (Figure S6). Even for hexamers, reaching such a large shift requires close contact, below $3.5 \mathrm{~nm}$ for the first peak and below $1.5 \mathrm{~nm}$ for the second. Allowing for possible systematic errors in the BEM simulations at very short distances, we can however conclude that a vast majority of the contacts are "intimate", with at most one intervening bilayer for the first one and practically no surfactant for the second. This conclusion is supported by the fact that the amplitude of the second mode decreases with increasing CTAB concentration in solution (presumably also leading to more surfactant being present on the NP surfaces).

\section{Chain statistics from TEM}

The time-resolved AS measurements yield the assembly rate $\mathbf{C}_{2}(t)$, but the method is indirect, so we confirm it by direct inspection of the individual objects. We have seen that the kinetics can be controlled by adjusting the surfactant concentration. In addition, we

have shown ${ }^{22}$ that the reaction can be arrested by increasing the CTAB concentration up to the millimolar range. After supplementing the dispersion with $10 \mathrm{mM} \mathrm{CTAC}$, the optical properties did not significantly change over one month, demonstrating the stabilization of the assemblies.

We used TEM to check for one system (bipyramids in $1 \mathrm{mM} \mathrm{MgSO} 4$ ) the aggregation 
state at the beginning of the process $(0 \mathrm{~h})$ and after 5 and 20 hours. Some images are shown in Figure S2b The results are displayed in Figure 9. Aside from the number of particles in aggregates of various sizes (9a), we are also interested in the relative position of the particles so we sort the interparticle contacts into tip-to-tip, tip-to-side and side-to-side (9b). From Figure 9a we extract $\mathbf{C}_{2}(t)=0.19,0.57$ and 0.69 at $t=0,5 \mathrm{~h}$ and $20 \mathrm{~h}$, respectively, in good agreement with the AS data.

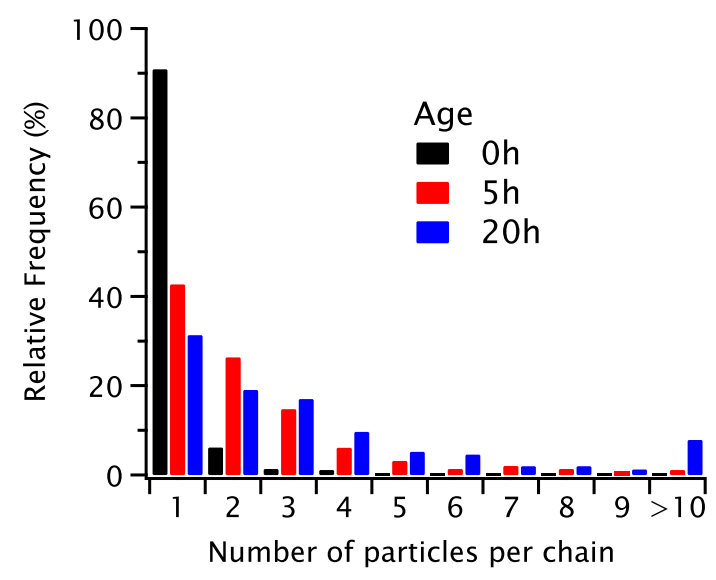

a)

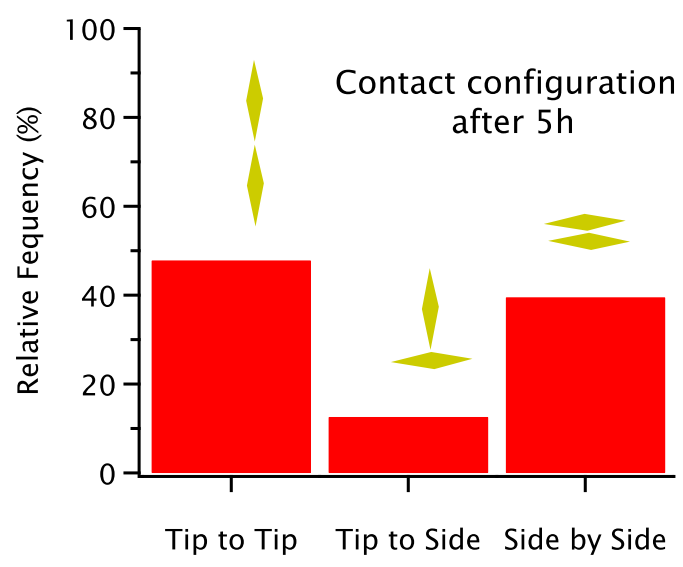

b)

Figure 9: a) Statistics of the aggregate size measured by TEM for three different times $(0 \mathrm{~h}$, $5 \mathrm{~h}$ and $20 \mathrm{~h}$ ) after the beginning of the process. Each count represents one object (isolated particle or aggregate) and we analyzed at least 400 objects for each time. b) Statistics of the contact distribution after 5 hours. Each count represents one contact and we analyzed 625 contacts.

The Carothers equation predicts a degree of polymerization $\bar{X}_{n}(t)=\frac{1}{1-\mathbf{C}_{2}(t)}$, i.e. values of $1.2,2.3$ and 3.2 after 0,5 and $20 \mathrm{~h}$, in excellent agreement with the experimental TEM results: 1.1, 2.3 and 3.3. Therefore, the second step of the assembly seems to follow a step-growth mechanism (both monomers and oligomers can self-assemble ${ }^{40}$ ), albeit with an apparent first-order kinetics, unlike for organic polymers, for which the kinetics is generally second or higher order. ${ }^{41}$ This unusual behavior is probably due to the rapid aggregation in the first step, which already brings the particles close together. 


\section{Particle interaction}

Direct information on the interparticle distance can be obtained by TEM. In conventional TEM the particles are deposited on a substrate and dried, so there is no guarantee that this distance is preserved. We therefore used LCTEM to measure the tip-to-tip and side-to-side distances between bipyramids in assemblies (see Figure S12), obtaining about $1 \mathrm{~nm}$ for the former and $2-3 \mathrm{~nm}$ for the latter, in rough agreement with the values inferred from the AS data for nanorods.

Not only the tip-to-tip distance is small, but the assemblies are very stable under centrifugation $^{22}$ and with respect to dialysis (see Figure S11), so the interaction is presumably quite strong. Using time-resolved LCTEM imaging we could characterize this interaction by analyzing the positional and orientational fluctuations of objects in an assembly. We studied a bipyramid-sphere dimer (see Figure 10a) and a heptamer of bipyramids (Figure 10b). For the former, we extracted from the images the center-to-center distance and the tilt of the bipyramid with respect to the center line; for the latter, we used the distance between the centers of the upper part, consisting of three particles, and the lower one, consisting of four particles, and the angle between the long axis of these objects. The raw data is presented in Figure S13. It should be noted that these objects were attached to the membrane of the observation cell at the upper extremity (dimer) or at the lower one (heptamer) but were otherwise free to swing around these points (see the Movies in Supporting Information).

We obtained 120 usable frames for the dimer and 202 for the heptamer. The histograms

of the distances and angles were fitted with a Gaussian function: $\exp \left(\frac{x-x_{0}}{\sigma}\right)^{2}$ yielding the standard deviation $\sigma$ and the associated uncertainty, represented in Figure 10c and d) as dots and error bars. For the dimer we also have a shorter segment (23 images) and we extract $\sigma$ as the standard deviation (point shown without error bars).

The interparticle distance varied by about $2 \mathrm{~nm}$. Surprisingly, the angle only fluctuates by about $25 \mathrm{mrad}\left(\right.$ about $1.5^{\circ}$ ), so the interaction is also extremely directional.

We emphasize that we do not analyze the absolute distance, but rather its fluctuations. 


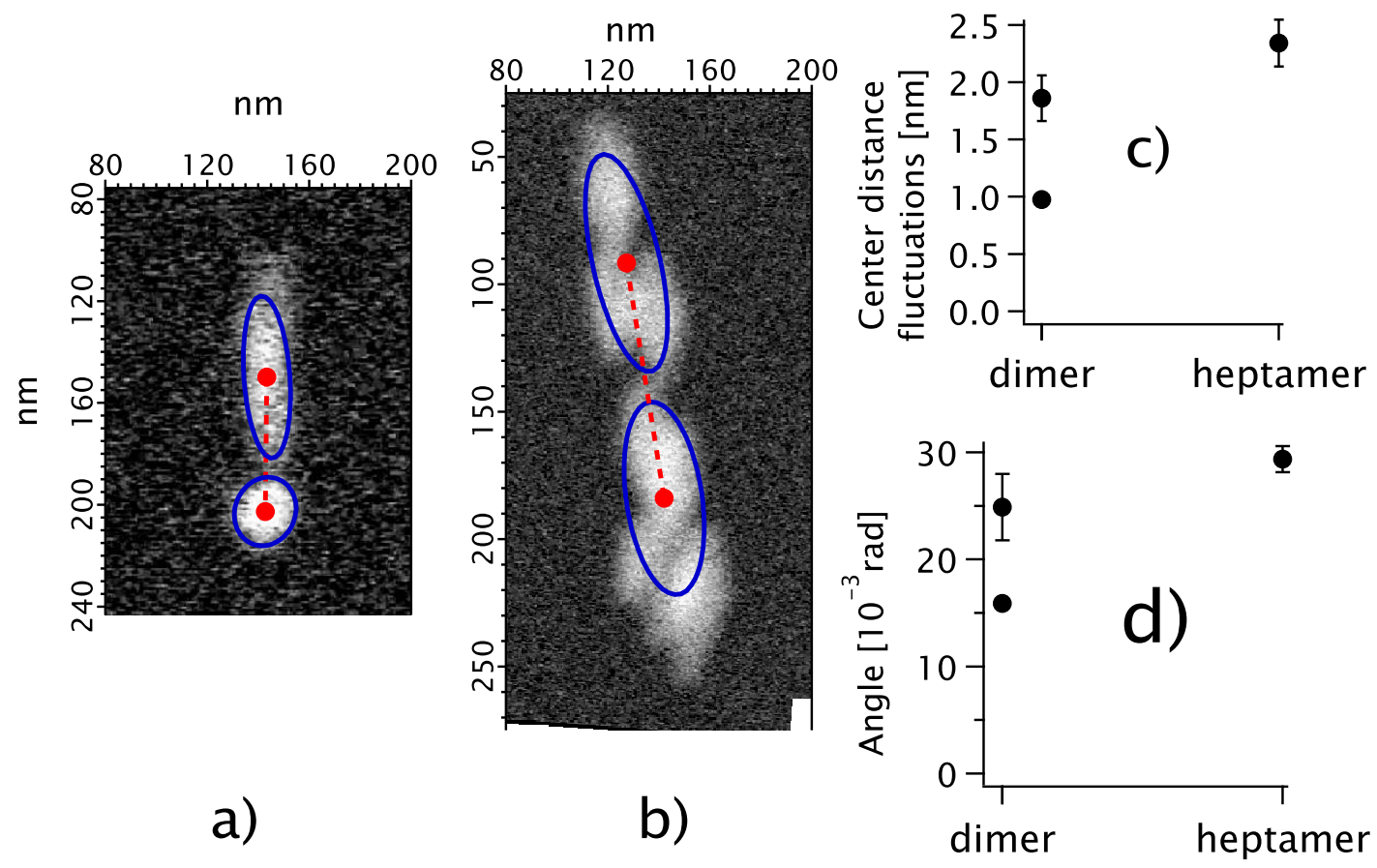

Figure 10: LCTEM images: a) Bipyramid-sphere dimer and b) heptamer. The particle outlines used to define the center of mass of the single particles or groups of particles on each side of the analyzed connection point are shown as blue solid lines. The center-to-center segments are shown as dashed red lines. Analysis: standard deviation of fluctuation amplitudes for c) the center-to-center distance and d) the interparticle angle. 
Configuration changes within the two particle groups would increase the fluctuation amplitude, so the values in Figure 10c are in fact upper boundaries for the amplitude of fluctuations in contact distance.

\section{Conclusions}

Let us summarize the results of the various techniques in a coherent picture of the assembly. The process is driven by reducing the CTAB concentration well below the CMC: immediately (a few minutes, at most) after this step large objects are present in the solution, as revealed by the small-angle increase in the static SAXS data (Figure 2). At this point, the UVVis spectrum is still unchanged, because the interparticle distances are above a few $\mathrm{nm}$, presumably imposed by the surfactant bilayers coating them. The chains, if present, do not have a well-defined spacing (we observe no peaks in the SAXS data).

The assembly can be simply described as the conversion from the initial species (isolated particles) to the final one (chains), but with an apparent first-order kinetics (see Figures 4 and 5 and the corresponding discussion). This conclusion holds very well for rods and spheres, but not for bipyramids, where the evolution is more complicated, both in terms of the number of species involved and of the time evolution.

The final objects exhibit both tip-to-tip and side-to-side assembly. While the side-to-side distance is large enough to accommodate two surfactant bilayers, as expected for CTABcovered objects merely coming into contact, the tip-to-tip distance is much smaller, so the surfactant must have been expelled from the interspace, leaving either one bilayer or essentially no surfactant. The interparticle distance is relatively well defined, with a peak in the SAXS structure factor (Figure 3).

The rate-limiting step of the reaction could be related to surfactant expulsion. This is supported by the apparently first-order character of the reaction, which is in apparent contradiction with an aggregation process, where the initial particles must first encounter 
each other, leading to (at least) second-order kinetics. To understand it, we must keep in mind that the AS spectra only change when the particles are in close proximity: the particles in the same population (described by $\mathbf{S}_{1}(\lambda)$ ) may very well be together in a loose clump; it is only when they get together and form chains that their spectrum shifts to $\mathbf{S}_{2}(\lambda)$. While the aggregation step is controlled by the CTAB concentration, the sulfate ions are involved in the formation of tip-to-tip contacts, in agreement with the conclusion of Abtahi et al. ${ }^{21}$ As to the kinetics, we show that it is apparently first-order, as opposed to the second-order behavior found by these authors.

Finally, we posit that such a two-step evolution, which can only be uncovered by combining AS with a structural technique (e.g. SAXS), may very well occur in other processes exhibiting an apparent first-order kinetics, for instance when Au NPs assemble due to the induction of an electric dipole at their surface. ${ }^{36}$

\section{Supporting Information Available}

The following files are available free of charge.

- Determination of the particle sizes, singular value decomposition method for the AS data, MNPBEM simulations of various particle assemblies, goodness-of-fit analysis of the AS kinetics, dialysis tests and analysis of the LCTEM videos. (PDF file)

- LCTEM video showing the fluctuations of the dimer discussed in the text. (AVI file)

- LCTEM video showing the fluctuations of the heptamer discussed in the text. (AVI file)

\section{Conflicts of interest}

There are no conflicts to declare. 


\section{Acknowledgement}

J. L. acknowledges financial support by the China Scholarship Council (CSC). We acknowledge SOLEIL for provision of synchrotron radiation facilities and we would like to thank Javier Perez and Thomas Bizien for assistance in using beamline SWING for the SAXS experiments. We thank Claire Goldmann for the preparation of some nanoparticle batches and for continuous experimental support.

\section{References}

1. Chen, L.; Su, B.; Jiang, L. Recent advances in one-dimensional assembly of nanoparticles. Chemical Society Reviews 2018,

2. Xi, C.; Marina, P. F.; Xia, H.; Wang, D. Directed self-assembly of gold nanoparticles into plasmonic chains. Soft Matter 2015, 11, 4562-4571.

3. Taylor, R. W.; Lee, T.-C.; Scherman, O. A.; Esteban, R.; Aizpurua, J.; Huang, F. M.; Baumberg, J. J.; Mahajan, S. Precise Subnanometer Plasmonic Junctions for SERS within Gold Nanoparticle Assemblies Using Cucurbit[n] uril "Glue". ACS Nano 2011, $5,3878-3887$.

4. Caswell, K. K.; Wilson, J. N.; Bunz, U. H. F.; Murphy, C. J. Preferential End-to-End Assembly of Gold Nanorods by Biotin-Streptavidin Connectors. Journal of the American Chemical Society 2003, 125, 13914-13915.

5. Wang, L.; Zhu, Y.; Xu, L.; Chen, W.; Kuang, H.; Liu, L.; Agarwal, A.; Xu, C.; Kotov, N. Side-by-Side and End-to-End Gold Nanorod Assemblies for Environmental Toxin Sensing. Angewandte Chemie International Edition 2010, 49, 5472-5475.

6. Lin, S.; Li, M.; Dujardin, E.; Girard, C.; Mann, S. One-Dimensional Plasmon Coupling 
by Facile Self-Assembly of Gold Nanoparticles into Branched Chain Networks. Advanced Materials 2005, 17, 2553-2559.

7. Shibu Joseph, S. T.; Ipe, B. I.; Pramod, P.; Thomas, K. G. Gold Nanorods to Nanochains: Mechanistic Investigations on Their Longitudinal Assembly Using $\alpha, \omega$-Alkanedithiols and Interplasmon Coupling. The Journal of Physical Chemistry B 2006, 110, 150-157.

8. Hamon, C.; Bizien, T.; Artzner, F.; Even-Hernandez, P.; Marchi, V. Replacement of CTAB with peptidic ligands at the surface of gold nanorods and their self-assembling properties. Journal of Colloid and Interface Science 2014, 424, 90-97.

9. Liu, K.; Nie, Z.; Zhao, N.; Li, W.; Rubinstein, M.; Kumacheva, E. Step-Growth Polymerization of Inorganic Nanoparticles. Science 2010, 329, 197-200.

10. Nie, Z.; Fava, D.; Kumacheva, E.; Zou, S.; Walker, G. C.; Rubinstein, M. Self-assembly of metalpolymer analogues of amphiphilic triblock copolymers. Nature Materials 2007 , $6,609-614$.

11. Hanske, C.; Tebbe, M.; Kuttner, C.; Bieber, V.; Tsukruk, V. V.; Chanana, M.; König, T. A. F.; Fery, A. Strongly Coupled Plasmonic Modes on Macroscopic Areas via TemplateAssisted Colloidal Self-Assembly. Nano Letters 2014, 14, 6863-6871.

12. González-Rubio, G.; González-Izquierdo, J.; Bañares, L.; Tardajos, G.; Rivera, A.; Altantzis, T.; Bals, S.; Peña Rodrguez, O.; Guerrero-Martnez, A.; Liz-Marzán, L. M. Femtosecond Laser-Controlled Tip-to-Tip Assembly and Welding of Gold Nanorods. Nano Letters 2015, 15, 8282-8288.

13. Herrmann, L. O.; Valev, V. K.; Tserkezis, C.; Barnard, J. S.; Kasera, S.; Scherman, O. A.; Aizpurua, J.; Baumberg, J. J. Threading plasmonic nanoparticle strings with light. Nature Communications 2014, 5, 4568. 
14. Fontana, J.; Charipar, N.; Flom, S. R.; Naciri, J.; Piqué, A.; Ratna, B. R. Rise of the Charge Transfer Plasmon: Programmable Concatenation of Conductively Linked Gold Nanorod Dimers. ACS Photonics 2016, 3, 904-911.

15. Klinkova, A.; Therien-Aubin, H.; Choueiri, R. M.; Rubinstein, M.; Kumacheva, E. Colloidal analogs of molecular chain stoppers. Proceedings of the National Academy of Sciences 2013, 110, 18775-18779.

16. Stewart, A. F.; Lee, A.; Ahmed, A.; Ip, S.; Kumacheva, E.; Walker, G. C. Rational Design for the Controlled Aggregation of Gold Nanorods via Phospholipid Encapsulation for Enhanced Raman Scattering. ACS Nano 2014, 8, 5462-5467.

17. Stewart, A. F.; Gagnon, B. P.; Walker, G. C. Forming End-to-End Oligomers of Gold Nanorods Using Porphyrins and Phthalocyanines. Langmuir 2015, 31, 6902-6908.

18. Yang, M.; Chen, G.; Zhao, Y.; Silber, G.; Wang, Y.; Xing, S.; Han, Y.; Chen, H. Mechanistic Investigation into the Spontaneous Linear Assembly of Gold Nanospheres. Physical Chemistry Chemical Physics 2010, 12, 11850.

19. Han, X.; Goebl, J.; Lu, Z.; Yin, Y. Role of Salt in the Spontaneous Assembly of Charged Gold Nanoparticles in Ethanol. Langmuir 2011, 27, 5282-5289.

20. Liu, K.; Resetco, C.; Kumacheva, E. Salt-Mediated Kinetics of the Self-Assembly of Gold Nanorods End-Tethered with Polymer Ligands. 2012, 4, 6574.

21. Abtahi, S. M. H.; Burrows, N. D.; Idesis, F. A.; Murphy, C. J.; Saleh, N. B.; Vikesland, P. J. Sulfate Mediated End-to-End Assembly of Gold Nanorods. Langmuir 2017,

22. Li, X.; Lyu, J.; Goldmann, C.; Kociak, M.; Constantin, D.; Hamon, C. Plasmonic Oligomers with Tunable Conductive Nanojunctions. The Journal of Physical Chemistry Letters 2019, 7093-7099. 
23. Ye, X.; Jin, L.; Caglayan, H.; Chen, J.; Xing, G.; Zheng, C.; Doan-Nguyen, V.; Kang, Y.; Engheta, N.; Kagan, C. R. et al. Improved Size-Tunable Synthesis of Monodisperse Gold Nanorods through the Use of Aromatic Additives. ACS Nano 2012, 6, 2804-2817.

24. Scarabelli, L.; Grzelczak, M.; Liz-Marzán, L. M. Tuning Gold Nanorod Synthesis through Prereduction with Salicylic Acid. Chemistry of Materials 2013, 25, 4232-4238.

25. Sánchez-Iglesias, A.; Winckelmans, N.; Altantzis, T.; Bals, S.; Grzelczak, M.; LizMarzán, L. M. High-Yield Seeded Growth of Monodisperse Pentatwinned Gold Nanoparticles through Thermally Induced Seed Twinning. Journal of the American Chemical Society 2017, 139, 107-110.

26. Chateau, D.; Liotta, A.; Vadcard, F.; Navarro, J. R. G.; Chaput, F.; Lermé, J.; Lerouge, F.; Parola, S. From gold nanobipyramids to nanojavelins for a precise tuning of the plasmon resonance to the infrared wavelengths: experimental and theoretical aspects. Nanoscale 2015, 7, 1934-1943.

27. Hanske, C.; González-Rubio, G.; Hamon, C.; Formentín, P.; Modin, E.; Chuvilin, A.; Guerrero-Martínez, A.; Marsal, L. F.; Liz-Marzán, L. M. Large-Scale Plasmonic Pyramidal Supercrystals via Templated Self-Assembly of Monodisperse Gold Nanospheres. The Journal of Physical Chemistry C 2017, 121, 10899-10906.

28. Kline, S. R. Reduction and analysis of SANS and USANS data using IGOR Pro. Journal of Applied Crystallography 2006, 39, 895-900.

29. Hohenester, U.; Trügler, A. MNPBEM-A Matlab toolbox for the simulation of plasmonic nanoparticles. Computer Physics Communications 2012, 183, 370-381.

30. Ahmad, N.; Wang, G.; Nelayah, J.; Ricolleau, C.; Alloyeau, D. Exploring the Formation of Symmetric Gold Nanostars by Liquid-Cell Transmission Electron Microscopy. Nano Letters 2017, 17, 4194-4201. 
31. Piffoux, M.; Ahmad, N.; Nelayah, J.; Wilhelm, C.; Silva, A.; Gazeau, F.; Alloyeau, D. Monitoring the Dynamics of Cell-Derived Extracellular Vesicles at the Nanoscale by Liquid-Cell Transmission Electron Microscopy. Nanoscale 2018, 10, 1234-1244.

32. Slyusarenko, K.; Abécassis, B.; Davidson, P.; Constantin, D. Morphology of Gold Nanoparticles Determined by Full-Curve Fitting of the Light Absorption Spectrum. Comparison with X-Ray Scattering and Electron Microscopy Data. Nanoscale 2014, 6, 13527-13534.

33. Funston, A. M.; Novo, C.; Davis, T. J.; Mulvaney, P. Plasmon Coupling of Gold Nanorods at Short Distances and in Different Geometries. Nano Letters 2009, 9, 1651-1658.

34. Shao, L.; Woo, K. C.; Chen, H.; Jin, Z.; Wang, J.; Lin, H.-Q. Angle- and Energy-Resolved Plasmon Coupling in Gold Nanorod Dimers. ACS Nano 2010, 4, 3053-3062.

35. Cohen, M. D.; Fischer, E. 588. Isosbestic Points. Journal of the Chemical Society (Resumed) 1962, 3044-3052.

36. Li, M.; Johnson, S.; Guo, H.; Dujardin, E.; Mann, S. A Generalized Mechanism for Ligand-Induced Dipolar Assembly of Plasmonic Gold Nanoparticle Chain Networks. 2011, 21, 851-859.

37. Janicek, B. E.; Hinman, J. G.; Hinman, J. J.; hyun Bae, S.; Wu, M.; Turner, J.; Chang, H.-H.; Park, E.; Lawless, R.; Suslick, K. S. et al. Quantitative Imaging of Organic Ligand Density on Anisotropic Inorganic Nanocrystals. Nano Letters 2019, 19, 6308-6314.

38. Richetti, P.; Kékicheff, P. Direct Measurement of Depletion and Structural Forces in a Micellar System. Physical Review Letters 1992, 68, 1951-1954.

39. Kékicheff, P.; Contal, C. Cationic-Surfactant-Coated Mica Surfaces below the Critical 
Micellar Concentration: 1. Patchy Structures As Revealed by Peak Force Tapping AFM Mode. Langmuir 2019, 35, 3087-3107.

40. Li, Z.; Wang, W.; Yin, Y. Colloidal Assembly and Active Tuning of Coupled Plasmonic Nanospheres. Trends in Chemistry 2020, 2, 593-608.

41. Odian, G. G. Principles of Polymerization, 4th ed.; Wiley-Interscience: Hoboken, N.J, 2004 . 
Graphical TOC Entry

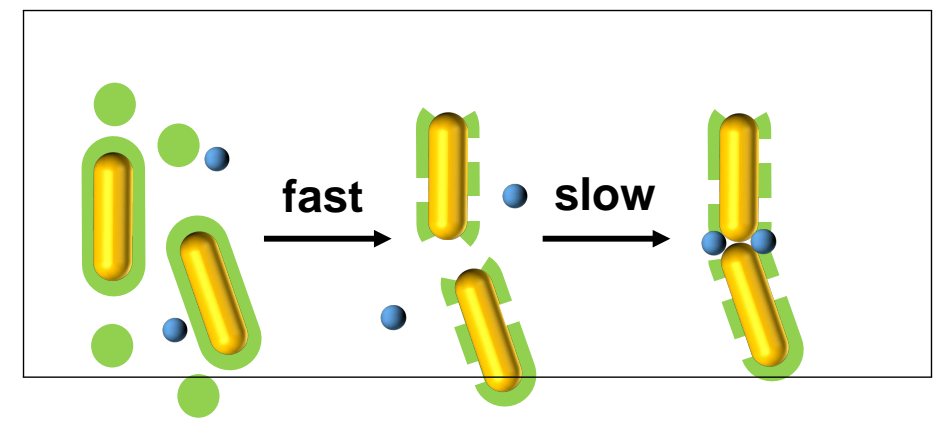

